\title{
La construcción del sistema de soberanía alimentaria y nutrición del Ecuador
}

\section{Fernando Rosero*}

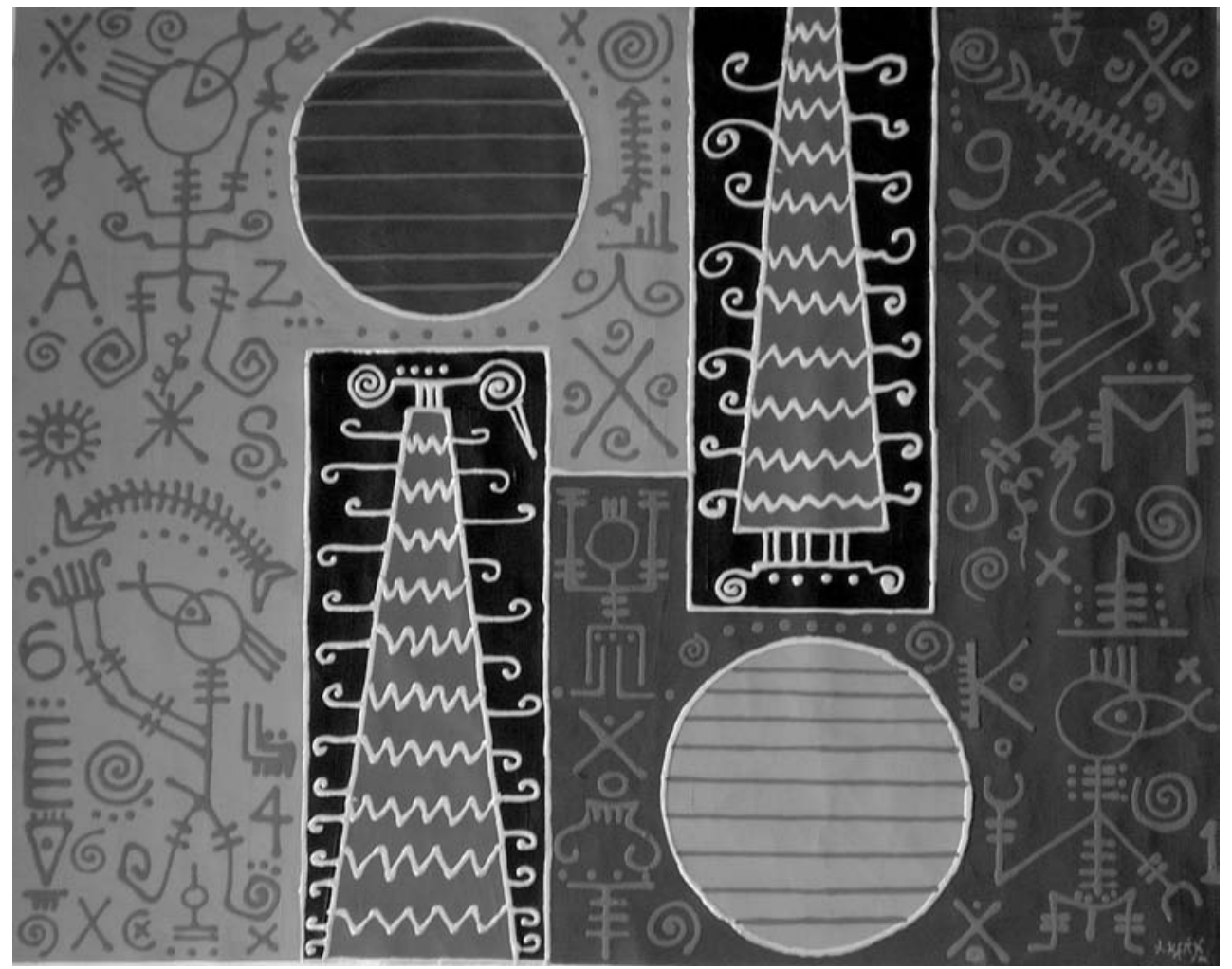

Fusiones. Técnica mixta sobre lienzo

El nuevo paradigma de la soberanía alimentaria surgió a mediados de la década de los noventa como propuesta de las organizaciones campesinas de América Central ante el proceso de desestructuración de las agriculturas familiares de la región. Si bien este proceso se profundi-

* El autor es sociólogo rural graduado en la Universidad de París III, profesor de la Escuela de Gestión para el Desarrollo Local Sostenible de la UPS-Q. Actualmente se desempeña como coordinador del Centro Andino para la Formación de Líderes Sociales (CAFOLIS). 
zó con la ayuda alimentaria de la cooperación internacional ante los huracanes y la violencia generados por la naturaleza y los humanos, ya se había dado por la presencia de productos alimentarios subsidiados provenientes de los países industrializados y vendidos localmente a bajos precios, configurando verdaderas estrategias de dumping.

La nueva propuesta de soberanía alimentaria tiene antecedentes en las políticas de seguridad alimentaria surgidas después de la Segunda Guerra Mundial, en Europa, frente al hambre desencadenada por esa gran conflagración. Se planteó, entonces, la necesidad de reconocer la universalidad del derecho a la alimentación, es decir, que todas las personas, de todos los sectores sociales puedan acceder, de manera permanente, a los alimentos necesarios. Con la creación de la Unión Europea en 1957, se consolidó la idea de que estos productos debían ser preferentemente nacionales o europeos para desarrollar las economías nacionales y para asegurar la soberanía del Estado, considerando a los alimentos como arma estratégica para la independencia de los Estados.

\section{Complejidad y multidimensiona- lidad de la soberanía alimentaria}

El nuevo paradigma de la soberanía alimentaria es complejo y multidimensional porque se plantea como una estrategia y una obligación del Estado, como lo reconoce el Art. 281 de la nueva Constitución Política de la República. Se trata de una de las estrategias clave del nuevo modelo de desarrollo económico, ambiental, social y cultural, junto con la propuesta de economía popular solidaria.

Desde esta perspectiva, la soberanía alimentaria involucra a todos los sectores de la economía, el primario, el industrial y el de servicios, pero también concierne a la producción de alimentos en la agricultura, la ganadería, la pesca artesanal, el procesamiento de alimentos, el intercambio, la comercialización, la distribución y el consumo de alimentos. Es decir que los sistemas alimentarios involucran a toda la población del campo y de las ciudades ya sea como productores de alimentos, como agentes de transformación, transporte, comercialización o simplemente como consumidores.

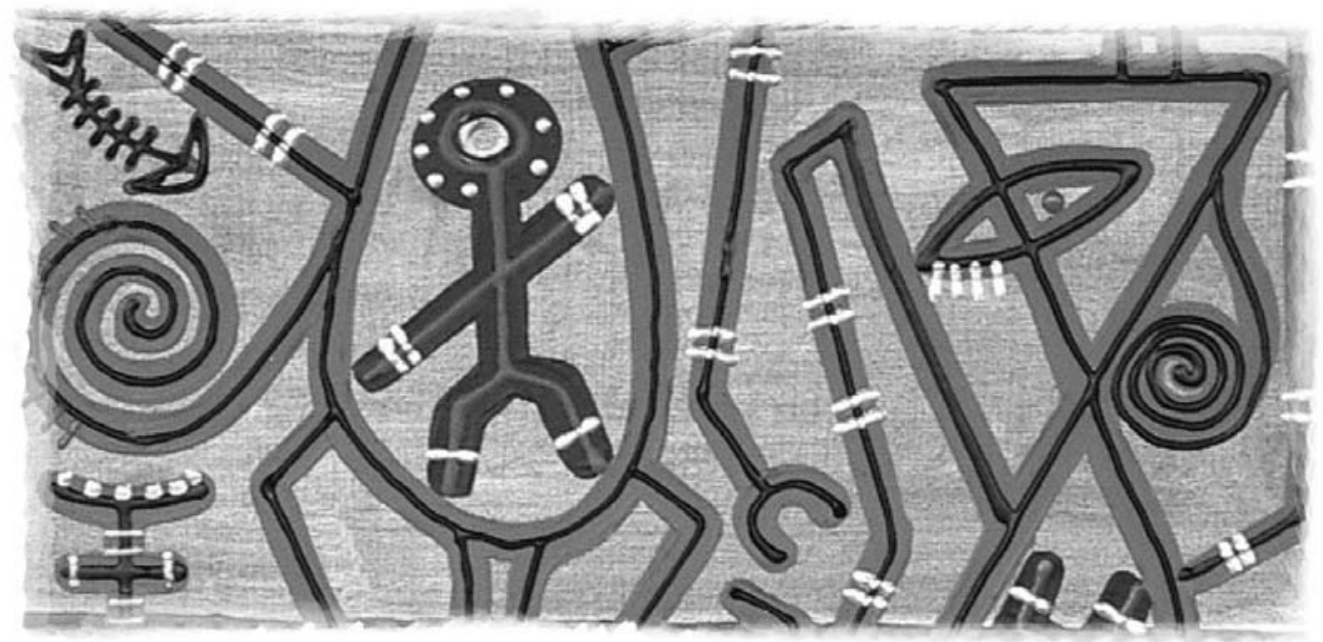

Reminiscencias. Detalle 
Por su carácter comprensivo la soberanía alimentaria en su dimensión institucional no puede ser competencia o coto de caza de un solo ministerio, sino que tiene naturaleza supra-ministerial, pues involucra la nutrición, la salud, la educación, el ambiente y por supuesto los diversos sectores de la economía. Brasil creó, en septiembre de 2006, la Cámara Interministerial a nivel del Estado Federal y las Cámaras Intersecretariales en los Estados de la federación para responder a este enfoque, pero sobre todo para dar salida a los celos institucionales y a las tendencias corporativas presentes en el sector público y en las culturas políticas latinoamericanas.

El médico y sociólogo brasileño Josué de Castro mostró el carácter engañoso de la idea que el hambre es un sino, un destino preestablecido o fatum, al poner en evidencia que es una construcción social generada por modelos de desarrollo de carácter inequitativo, como el modelo brasileño de industrialización de mediados del siglo pasado. Pero además, Josué de Castro tuvo el mérito de hacer visibles las interrelaciones entre las deficiencias en la nutrición, la salud de las personas, su desarrollo físico e intelectual, y el desarrollo de las naciones (Maluf, 2008). Por ello, de Castro prefirió hablar de la seguridad alimentaria y nutricional, calificativo posteriormente recogido por la FAO y por el sistema brasileño de seguridad alimentaria.

\section{La centralidad de la agricultura familiar}

La diversidad de modelos de crecimiento hacia afuera se sustentó en la explotación de grandes extensiones de tierra para la exportación, primero del cacao, y posteriormente de banano. Las reformas agrarias de 1964 y 1973 distribuyeron tierras marginales, no afectaron el monopolio de la tierra y re-funcionalizaron los sistemas productivos rurales a las nuevas demandas del mercado. La ley agraria de 1994 cerró definitivamente el ciclo de la reforma agraria y creó las condiciones para el desarrollo de la agroindustria moderna de flores, brócoli, espárragos, etc., orientada hacia la exportación.

Los modelos de crecimiento hacia afuera sometieron a las economías campesinas a los intereses del capital financiero de las empresas importadoras de agroquímicos, de los exportadores y de los comerciantes locales y nacionales. La relación con la parcela y las comunidades locales permitieron la venta de sus productos alimentarios y de la fuerza de trabajo por debajo de su costo de producción. En los últimos años, las grandes empresas de alimentos orientadas hacia el mercado interno y hacia la exportación han controlado a las agriculturas familiares y a las economías campesinas a través de lo que se ha dado en llamar la agricultura por contrato. Este es el sistema productivo por el cual las pequeñas y medianas unidades producen alimentos que posteriormente son procesados por las grandes empresas de alimentos, como PRONACA, la cual se abastece del maíz vendido a bajo precio por las economías campesinas controladas a través del aprovisionamiento de créditos, semillas, fitosanitarios y asistencia técnica.

El nuevo modelo de desarrollo propone mudar del predominio del agro-negocio para la exportación a la centralidad de la agricultura familiar y de la economía popular solidaria. Es decir, pasar del crecimiento hacia afuera, en el cual el valor generado localmente se traslada a las grandes empresas de bienes y servicios, a las ciudades, hacia los centros de desarrollo y de éstos hacia las grandes metrópolis de los países industrializados, hacia un desarrollo territorial endógeno en el cual el valor generado localmente se

El nuevo paradigma de la soberanía alimentaria es complejo y multidimensional porque se plantea como una estrategia y una obligación del Estado. 
reinvierta localmente en la producción de bienes y servicios, mejorando la calidad de vida de los pequeños y medianos productores y del conjunto de la población ecuatoriana.

En esta perspectiva, el Art. 281 de la nueva Constitución Política de la República, CPR, vincula el derecho de los pueblos a alimentarse, es decir, al autoabastecimiento, a la producción de las pequeñas y medianas unidades agrícolas, pecuarias y pesqueras, en las cuales predomina la mano de obra familiar, el uso de tecnologías amigables con el ambiente, y la satisfacción de las necesidades de la familia y de las comunidades locales. Este enfoque se sustenta en el rol histórico de abastecimiento del mercado interno jugado por este tipo de unidades de producción. Si bien ha caído en los últimos años la participación de las pequeñas y medianas unidades de producción en el abastecimiento de granos, carnes, frutas y legumbres, en la actualidad representa todavía alrededor del 40\% contra el 65\% en los años sesenta.

Pero esta no es la única función de las economías campesinas, ellas también se caracterizan por su enorme capacidad de absorción de mano de obra, es decir por la generación de empleo e ingresos. En la actualidad se afirma con insistencia la multifuncionalidad de la economía campesina y se pone énfasis en la utilización de tecnologías amigables con el medio ambiente en el contexto de una creciente preocupación por el calentamiento global y los impactos de los agrotóxicos en la salud humana.

Así por ejemplo, el Art. 104 de la Constitución de la Confederación Helvética afirma que:

“1. La Confederación vela para que la agricultura, por medio de una producción que responda a la vez a las exigencias del desarrollo sostenible y a las del mercado, contribuya sustancialmente a:

a. la seguridad del aprovisionamiento de la población;

b. la conservación de los recursos naturales y al mantenimiento del paisaje rural; c. la ocupación descentralizada del territorio.

2. Como complemento a las medidas de ayuda mutua que razonablemente se puede exigir de la agricultura y si fuere necesario derogando el principio de libertad económica, la Confederación promueve las unidades de producción campesinas que cultivan el suelo.

3. Ella concibe las medidas de manera que la agricultura responda a sus múltiples funciones. Sus competencias y tareas son sobre todo las siguientes:

a. ella complementa el ingreso campesino por medio de pagos directos a fin de remunerar equitativamente los servicios prestados, con la condición de que el productor presente la prueba de que satisface las exigencias de carácter ecológico;

b. ella promueve, por medio de iniciativas de carácter económico, las formas de producción, particularmente, amigables a la naturaleza y respetuosas del ambiente y de los animales;

c. ella legisla sobre la declaración del origen, de la calidad, de los métodos de producción y de los procedimientos de transformación de los productos alimenticios;

d. ella protege el ambiente contra los atentados vinculados a la utilización abusiva de abonos, de productos químicos y de otros productos auxiliares;

e. ella puede promover la investigación, la difusión y la formación agrícola y otorgar ayudas a las inversiones;

f. ella puede legislar sobre la consolidación de la propiedad rústica rural.

4. Para estos fines ella se obliga a orientar especialmente los créditos agrícolas, así como también los recursos generales de la Confederación"2.

Los campesinos y productores familiares ecuatorianos insisten, con razón, en la necesidad de que el Estado les de un tratamiento diferenciado en créditos, pero también en aranceles y en asesoría técnica. 


\section{Acceso a los factores de producción}

Uno de los pilares fundamentales de la nueva política de soberanía alimentaria es el acceso a la tierra, al agua y al capital, es decir a los factores de producción.

La nueva ley marco de soberanía alimentaria prevé la elaboración y publicación posterior de seis leyes conexas, entre ellas una ley de tierras y territorios, la ley de aguas y la ley de la banca pública.

La CPR y la ley marco ya contemplan créditos para los pequeños y medianos productores en condiciones especiales, pero sin duda se requiere de un nuevo instrumento legal en el cual se defina las reglas de juego para que este tipo de unidades de producción accedan al crédito para capital de operación, pero también para activos fijos.

La herencia colonial de monopolio de las tierras no fue substancialmente modificada ni por la llamada ley de manos muertas de la Revolución Liberal -la cual facilitó la transferencia de dominio de algunos terratenientes al Estado y de éste a arrendatarios vinculados al sector- ni por las reformas agrarias de los años sesenta y setenta. El tímido proceso de democratización de las tierras empujado por las reformas agrarias fue rápidamente parado a fines de la dictadura militar (1979), y con la ley agraria decretada en el período del Arq. Sixto Durán Ballén. Las reglas de juego neoliberales permitieron una rápida reconcentración de la tierra bajo la figura de empresas agrícolas, la mayoría de ellas dedicadas a la exportación. En la actualidad, el coeficiente de Gini indica que el grado de concentración de las tierras en Ecuador es de 0,80; es decir, uno de los más altos de América Latina y por ello la CPR tiene fundamentos sólidos para plantear el tema de la redistribución de la tierra mediante la conformación de un Fondo de Tierras. La nueva ley de soberanía alimentaria proporciona nuevos detalles sobre la función ambiental y social de las tierras, pero envía su tratamiento a la nueva
Asamblea Nacional, pues prevé un plazo de 365 días para su tratamiento.

De igual forma, la nueva ley de soberanía alimentaria prevé la elaboración de una nueva ley de aguas sustentada en la gestión social y en las prelaciones preescritas en la nueva constitución.

\section{La agroecología, una opción de vida}

La opción por la agricultura familiar está vinculada estrechamente a la opción por la agroecología, no sólo como tecnología sino sistema de vida, en el cual se articulan las dimensiones: económica, ambiental, socio-cultural y técnica.

La agroecología se sustenta en el reconocimiento de la persona y de los grupos humanos como parte del cosmos y de la naturaleza, no para dominarla y explotarla sino para interactuar con ella en un repetido proceso de re-alimentación del suelo (de los micronutrientes), de la agrobiodiversidad, pero también de las identidades personales, familiares y colectivas.

Partiendo de los suelos desgastados, legados por la "revolución verde", la producción agroecológica es rentable a partir de los cuatro o cinco años en los que se logra recuperar la fertilidad de los suelos por medio del abonamiento con materia orgánica y la optimización del uso del agua a través del riego por aspersión o del riego por goteo.

Como lo muestra la experiencia de los granjeros de Toacaso, Cotopaxi, la producción rotativa y permanente de legumbres en camas de $0,80 \mathrm{~cm}$ de ancho por $20 \mathrm{~m}$ de largo, genera un ingreso bruto de 2.000 dólares mensuales y un ingreso neto de alrededor de 1.000 dólares mensuales. Esto ha permitido el mejoramiento de la calidad de vida de las familias de estos granjeros en cuanto a la alimentación, la vivienda, la educación y los medios de transporte propio.

La producción agroecológica, como lo afirman sus actores, permite la reintegración familiar y evita la emigración interna e internacional. 


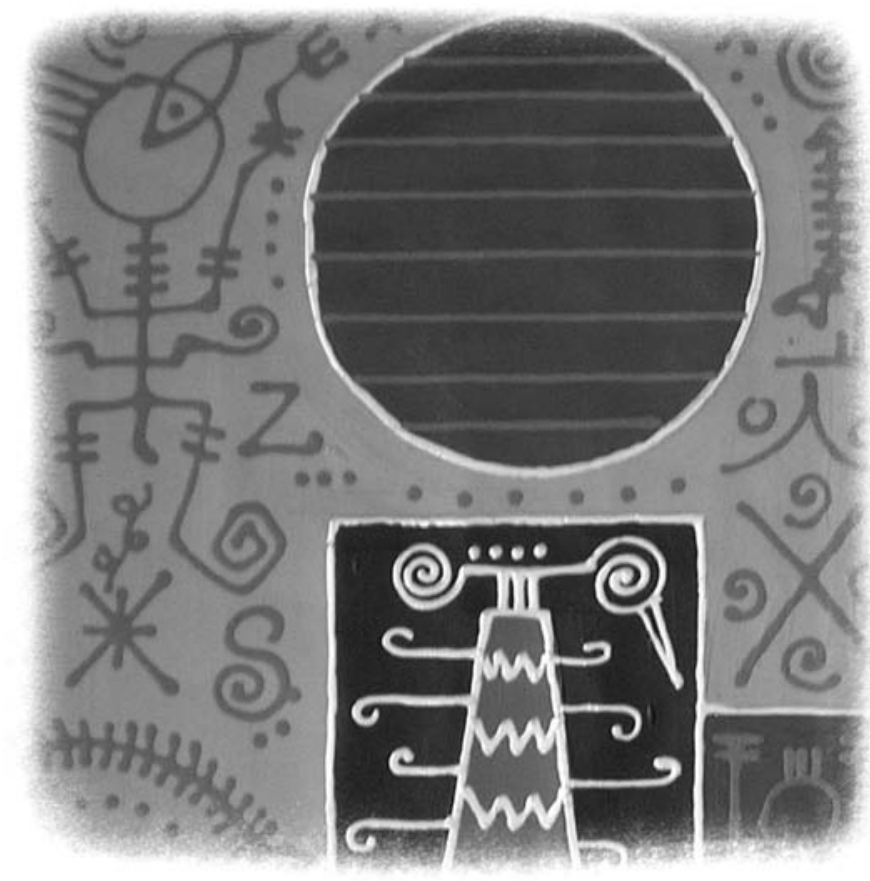

Fusiones. Detalle

En la actualidad hay en el país alrededor de tres mil unidades de producción agroecológicas y muy pocas orgánicas, en sentido estricto, porque la mayoría de ellas utilizan semillas provenientes de grandes empresas agro-alimentarias de Europa, Estados Unidos y Canadá. Lo ideal sería dar el paso a la producción de semillas y plántulas a nivel local a partir de la agro-biodiversidad local.

La difusión de la agro-ecología, la multiplicación de estas experiencias locales y la construcción de la política de Estado respectiva demandan el intercambio de experiencias; la articulación de las redes locales y regionales en una gran red nacional; el cambio institucional y la elaboración y ejecución de programas estratégicos que articulen proyectos y programas de tipo sectorial.

\section{El desafío de los transgénicos}

Las empresas transnacionales de alimentos pregonan las bondades de los transgénicos en relación al incremento de la productividad y de las utilidades, la inocuidad de los productos y la irrelevancia de sus impactos sobre el suelo y los sistemas de vida. En realidad, investigaciones realizadas en Argentina y Brasil muestran que los beneficios económicos altos se dan en los periodos de introducción de los cultivos de soja, pero que estos tienden a desaparecer una vez consolidado el paquete tecnológico y el control ex ante y ex pos por parte de las empresas transnacionales. Más todavía los estudios realizados en Canadá muestran los impactos contaminantes de los transgénicos en la biodiversidad y en la salud humana.

Detrás del negocio de los transgénicos está el interés por el control de la vida por parte de los grandes grupos agroalimentarios que buscan apoderarse de los genomas de plantas, animales e inclusive humanos, por medio de las patentes y otras formas de propiedad intelectual, a fin de utilizarlos en combinaciones genéticas a ser vendidas en el mercado, en paquetes tecnológicos que incluyen semillas, fertilizantes, fitosanitarios y asistencia técnica.

En este contexto se entiende la importancia estratégica del Art. 401 de la nueva CPR en que "Se declara al Ecuador libre de cultivos y semillas transgénicas". Sin embargo, y por presión de un grupo de empresarios en el mismo artículo se afirma que "Excepcionalmente y sólo en caso de interés nacional debidamente fundamentado por la Presidencia de la República y aprobado por la Asamblea Nacional, se podrá introducir semillas y cultivos genéticamente modificados".

\section{En busca de una nueva institucionalidad}

En su estudio sobre la gobernanza, Pierre Calame afirma que a menudo se quiere cambiar el 
Estado y las sociedades con ideas de ayer e instituciones de anteayer (Calame, 2008). Esta idea me ha rondado en la mente a lo largo del proceso de formulación de la norma constitucional y de la elaboración de la ley marco de soberanía alimentaria, pues el nuevo paradigma es poco conocido en el país, pero sobre todo por la dificultad de pensar los alimentos más allá de la producción agropecuaria y pesquera, a la luz del enfoque de la complejidad en el cual se articulan varios sectores y dimensiones, como ya se vio más arriba.

Este tipo de dificultad también está presente en el proceso de elaboración de la institucionalidad de la soberanía alimentaria pues hay quienes piensan que las nuevas demandas pueden ser atendidas por el Ministerio de Agricultura, Ganadería y Pesca, MAGAP re-estructurado o por un Comité creado especialmente para el caso, en el cual estén presentes representantes del Estado y de la sociedad civil, y cumpla las funciones de planificación participativa de la política pública, de ejecución y de seguimiento y evaluación. En los últimos meses ya se ha visto la posición de algunos dirigentes de las organizaciones sociales que conciben la soberanía alimentaria como una política intersectorial y supra-ministerial a funcionar a través de un Consejo Nacional, de la Conferencia Nacional y de un Gabinete interministerial.

Este debate se refleja en las diferentes versiones de la ley de soberanía alimentaria, especialmente en las elaboradas por la Comisión especializada de salud y ambiente de la Comisión Nacional de Legislación y Fiscalización.

Para precisar la nueva institucionalidad es necesario definir el Sistema de soberanía alimen- taria, sus componentes y funciones, confirmando los avances institucionales respecto del Consejo consultivo, como el responsable de la formulación, seguimiento y evaluación de la política pública de soberanía alimentaria, y de la Conferencia, como un espacio de deliberación pública con la participación de amplios sectores sociales. Pero también es indispensable definir claramente el Gabinete interministerial como espacio de coordinación de programas y políticas al interior del ejecutivo para la construcción del nuevo sistema de soberanía alimentaria, y como medio para superar los celos institucionales y las visiones corporativas propias de las culturas políticas latinoamericanas.

\section{Bibliografía}

- ASAMBLEA NACIONAL CONSTITUYENTE: Constitución Política de la República del Ecuador, aprobada el 24 de julio 2008 y publicada el 20 de octubre 2008.

- CALAME, Pierre: Hacia una revolución de la gobernanza. Reinventar la democracia, CEN, UASB, CAFOLIS, Quito, 2008.

- COMISIÓN ESPECIALIZADA DE SALUD Y AMBIENTE DE LA COMISIÓN NACIONAL DE LEGISLACIÓN Y FISCALIZACIÓN: Ley orgánica del régimen de soberanía alimentaria, Quito, febrero, 2009.

- CONFEDERACIÓNi HELVÉTICA: Constitution, janvier, 2008

- MALUF, Renato: La seguridad alimentaria y nutricional. Un enfoque de derecho y de soberanía, Quito, Editorial Silva, CAFOLIS, diciembre, 2008.

1 Este artículo fue escrito en febrero 2009, entre el primero y el segundo debate de la Comisión Nacional de Legislación y Fiscalización sobre la ley marco del régimen de soberanía alimentaria.

2 Ver la sección de sistemas alimentarios sostenibles del portal CAFOLIS: www.cafolis.org 